\title{
A Controller for Electric Sunroofs Based on Tablet PCs Using CAN Diagnosis
}

\author{
Xiao Chen ${ }^{1,}$, Feng Luo ${ }^{2, b}$ \\ ${ }^{1}$ Clean Energy Automotive Engineering Center, School of Automotive Studies, Tongji University, \\ Shanghai 201804, China \\ ${ }^{2}$ Clean Energy Automotive Engineering Center, School of Automotive Studies, Tongji University, \\ Shanghai 201804, China \\ aseancx3@gmail.com, bluo_feng@tongji.edu.cn
}

Keywords: Sunroof; Tablet PC; Unified Diagnostic Service; Controller Area Network

\begin{abstract}
Traditional methods of sunroof operations involve controllers with mechanic or electric buttons, which produce noises and lack flexibility. This paper devised a controller for electric sunroofs with CAN-USB converters and tablet PCs. The CAN-USB converter was implemented to bridge communication between tablet PCs and sunroofs. The tablet PC served as a control interface and an application was developed to control sunroofs by using the UDS protocol. A C language compiler was embedded in to support customized operations. This approach provides functionality of reprogramming for sunroof operations and offers sound-proof actions.
\end{abstract}

\section{Introduction}

Automobile sunroofs allow air to enter the passenger compartment. They may be manually operated or motor driven, while this article focuses on the latter[1]. Electric sunroof systems are usually cable driven by a motor and features some form of sliding operations, such as opening, closing and tilting.

The structure of a typical sunroof consists of four subassemblies: glass and sunshade assembly, drive mechanism, motion mechanism and frame assembly. A sunroof controller conducts motions of glasses or sunshades in a sunroof[2]. Generally speaking, a dedicated device, furnished with several mechanic buttons and a dot matrix LCD (Liquid Crystal Displayer), is designed for a certain type of sunroof. Operators could control the sunroof by pressing different buttons with the dot-matrix LCD displaying information, which is not user friendly. Besides, mechanic buttons would also introduce noise to this operation procedure, which disturbs the operator from identifying faults of sunroofs.

In this study, a tablet PC is utilized as the controlling interface to avoid noise. A CAN-USB converter was devised to exchange information between the tablet PCs and sunroofs. A control strategy based on UDS[3]-[5] on CAN was designed to manipulate sunroofs.

\section{System Description}

The whole system consists of three parts, including a tablet PC which provides controlling interface, a gateway and a sunroof with a motor mounted. A Windows 8 tablet PC is employed as the software platform provider, which offers users a noiseless and rich user interface. Owing to the lack of a CAN interface, the tablet PC is not directly connected to the CAN network. Thus, a CAN interface is developed to solve this problem. The CAN-USB converter, providing convenience on plug, high data rate and low cost[6], consists of microprocessors, a USB communication chip, and CAN transceivers with isolating circuits.

\section{Hardware Design}

The sunroof includes an electronic control module to provide CAN bus communication, supporting sunroof control via CAN bus. A CAN-USB converter is developed to interpret USB bus frames into CAN bus ones and vice versa, which supports different baud rates $(125 \mathrm{~Kb} / \mathrm{s}, 250 \mathrm{~Kb} / \mathrm{s}$, 
$500 \mathrm{~Kb} / \mathrm{s}$, and $1 \mathrm{Mb} / \mathrm{s}$ ) for the converter to communicate with diverse control modules.

The behavior and configuration of the converter can be controlled or set programmatically via USB bus. Configurations include baud rate, sample point, message transmission and reception behaviors.

This design uses a MCF51JM128 microchip, produced by Freescale, as a USB microcontroller. The MCF51JM128 incorporates a full-speed USB controller, fully compliant with USB specifications 1.1 and 2.0, and a CAN controller, which makes it qualified to be a converter. A CAN transceiver with electric isolation is used to protect the module when connecting to a CAN bus.

\section{Software Design}

The software consists of six modules, which are user interface, UDS on CAN, control strategy, security, USB driver and configuration file. The software architecture is shown in Fig. 1.



Fig. 1 Software architecture

The software architecture provides flexibility for different control modules. A configuration file unit is used to save configurations of different modules while a control strategy unit can adjust control methods between different control modules in sunroofs. A UDS on CAN unit is implemented to communicate with control modules of sunroofs, which can be substituted by other UDS implementations such as UDS on LIN (Local Interconnect Network) for further extension.

A unified user interface unit is designed to provide a universal interface for different kinds of electric sunroofs. The usual operations of sunroofs consist of normal opening, normal closing, normal tilting, express opening, express closing, express tilting, initialization, and thermal protection deactivation. Apart from those, moving a sunroof glass or shade to a certain position may also be required.

Control Strategy. For sunroof motors, the usual actions are open, close, tilt, initialization and thermal protection deactivation. Target sunroof motors are controlled by UDS on CAN. Those five actions can be represented by RoutineControl[3] services defined in UDS with different parameters, e.g. 0x31 0x00 0x01 represents opening sunroof glass, 0x31 0x00 0x02 represents closing sunroof glass, where 0x31 means executing service RoutineControl, while 0x00 0x01 and 0x00 0x02 are two parameter identifications.

Each action can be regarded as a group of commands, e.g. firstly, executing a command to enter diagnostic session for the sunroof control module, secondly, starting the routine, and finally, checking routine result. A check result routine is added to formulate a loop to make sure commands being properly executed. Fig. 2 demonstrates a workflow of a command.

Each command can be divided into two parts, firstly start, then wait and check its result periodically. This is the typical situation of UDS services. The sunroof acts as a server to receive and implement the requested services while the tablet PC serves as a client to generate and deliver those requests. 


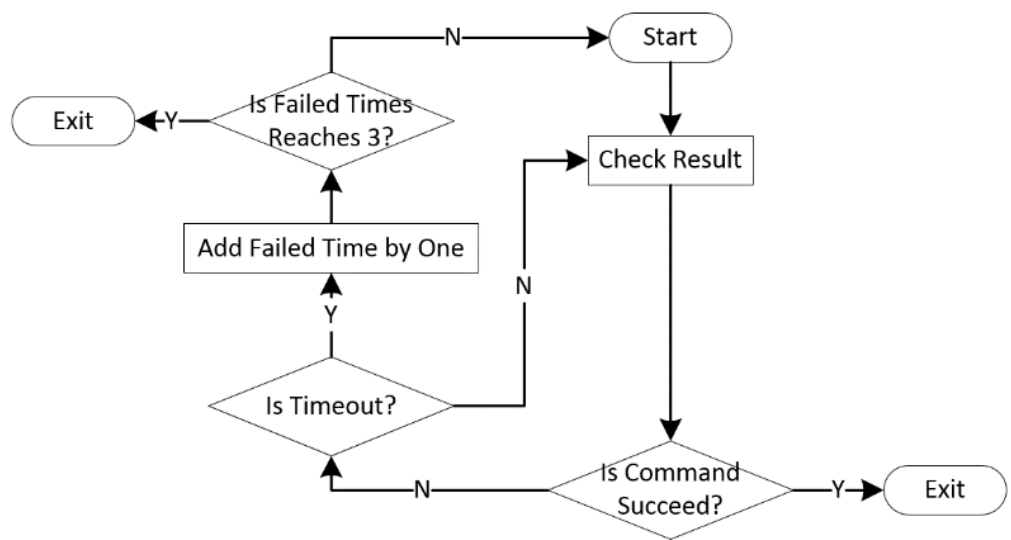

Fig. 2 Control strategy for an action

A command is started by transmitting a UDS request service to the sunroof electronic module. After the module received the request, it gives a response to the client to indicate whether this service has been executed successfully or not. If not, the request will be repeated 2 more times at the most.

Command Match. As this system is designed to control multiple kinds of sunroof motors, an action must be adjustable to match diverse motors. The system separates command implementations and command lists for each action. Thus, a command can have different contents when applied to different motors. To make the fullest use of codes, a command is shared across actions. This is done by loading command list and parameter list before executing commands, reflected by Fig. 3 .

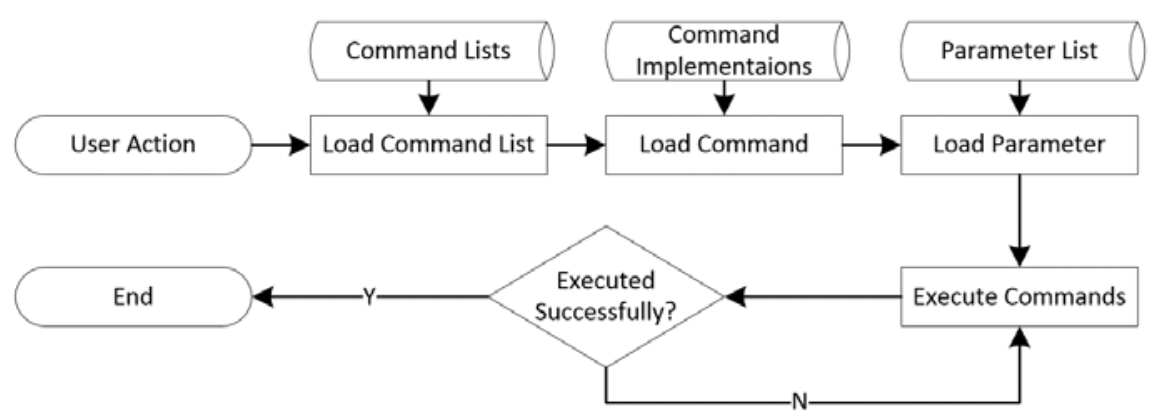

Fig. 3 Match actions and commands

Once a user action has been captured by the interface unit, the system executes a group of corresponding commands with four steps: first, loading corresponding command list; second, loading matched commands; third, loading parameters defined by the action; last, transmitting the commands in sequence and checking executing results.

Embedded C Compiler. Commands are implemented using C language, and can be edited and compiled in this system dynamically. A C compiler and an editing environment are embedded in the system, which offers a familiar programming environment for UDS software engineers. This allows operators change command behaviors whenever they want. The system uses a project configuration file to manage command implementations as well as another file to manage command lists, parameter lists and other configurations.

Each time a project configuration file being loaded, the system uses the embedded $\mathrm{C}$ compiler to build it. This sacrifices no execution time and offers flexibility for command implementations.

UDS Service. UDS services are implemented in this system. The tablet PC acts as a client to request a sunroof, the server, to execute corresponding services. The UDS services used in the systems are listed in Table 1. 
Table 1 UDS services used

\begin{tabular}{lll}
\hline SN. & Service Name & Functionality \\
\hline 1 & DiagnosticSessionControl & Enable different diagnostic sessions in the server \\
2 & ReadDataByIdentifier & Request data record from a server \\
3 & RoutineControl & Start or stop a routine, or request routine results \\
\hline
\end{tabular}

Three services have been used to fulfill the implementation: DiagnosticSessionControl for session control, ReadDataByIdentifier for reading data records, RoutineControl for routine control.

User interface. Fig. 4 shows the user interface. Two knobs handle general move operations, one for a glass and the other for a shade. A knob consists of several gears, whose count and value can be verified separately. A drop-down list is incorporated to select different kinds of sunroofs. When the selected sunroof changes, a check is done to verify whether the selection matches the connected sunroof or not, which is implemented by reading the data record in the connected sunroof and comparing it with the record in the database.

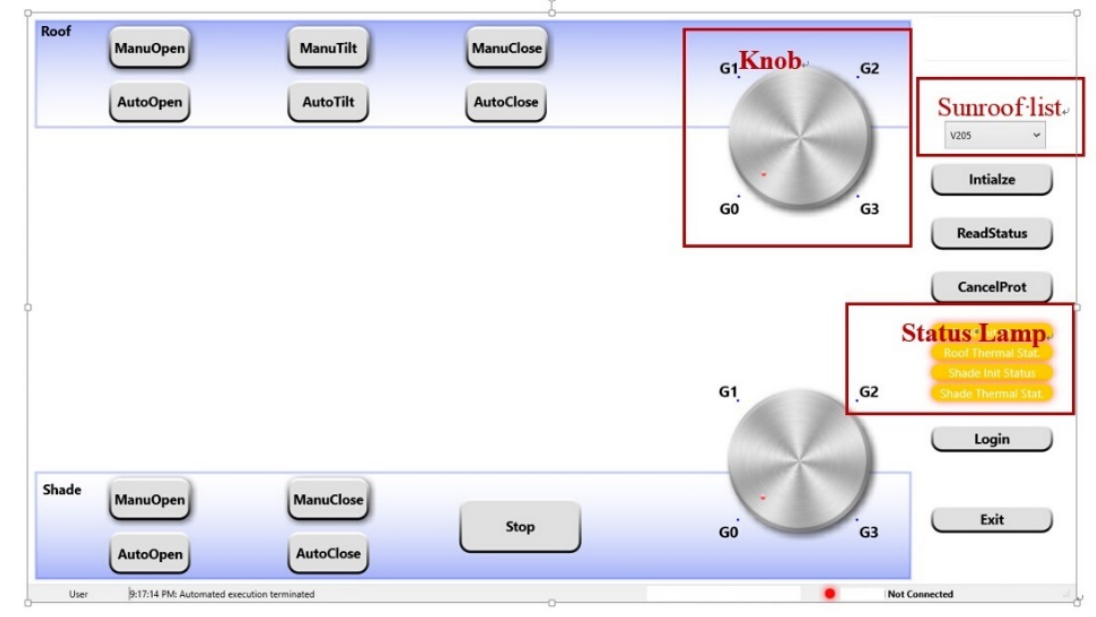

Fig. 4 Control interface

\section{Actions}

a) Normal open/close/tilt. A normal operation means an action required to be stopped when an actuator is released. Take a button designed for executing a glass normal open operation for example, the glass moves towards open direction when the button is pressed down, and stops when released.

b) Express open/close/tilt. An express operation means an action required to be executed when an actuator is activated, while releasing the actuator will not affect the ongoing action. Take a button designed to execute glass express open operation for example, the glass moves towards open direction when a button is pressed down, and will not stop even if the button is released.

c) Move to a certain position. A knob is used to activate this action. The count and value of gears for a knob can be changed in the 'Edit Command' window.

d) Stop. A stop button is necessary for stopping a mal-operation or for emergency. This operation stops a glass or a shade immediately. The motor monitors a periodic CAN frame to keep itself in a running state. If this frame is absent for more than one periodic interval, it stops. UDS services are not suitable for emergency stop as these services are executed in sequence. Thus, a stop service may be blocked by a previous one. A 100ms-period frame is chosen to keep the motor running.

e) Initialization \& thermal protection deactivation. In this system, RoutineControl services are used to provide these functionalities.

\section{Results}

The implemented system is as Fig. 5 shows. The sunroof motor is supplied by a power source of $12 \mathrm{~V}$. The tablet PC connects the CAN-USB converter with a USB cable, and the converter bridges 
the motor and the tablet PC.

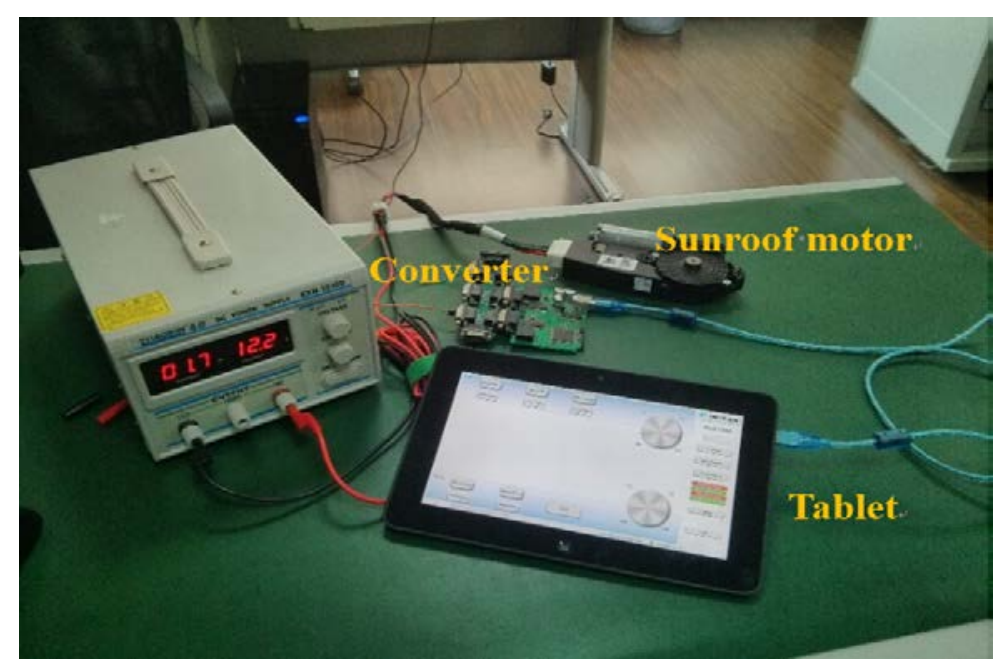

Fig. 5 Picture of the system

A controller based on a Windows 8 tablet PC was developed for general electric sunroofs. Express/normal operations of open, close, and tilt, or initialization, thermal protection deactivation have been implemented using diagnosis over CAN protocol. Also, a stop action has been added which stops the motor in 100 milliseconds whichever state the motor is in. An initialization operation can be performed in 40 seconds. In addition, a C compiler and code editor were embedded to provide flexibility for diverse sunroofs.

\section{Conclusion}

This study developed a sunroof controller based on tablet PCs with a CAN-USB converter. The controller is a user-friendly instrument which performs operations quickly and conveniently. A UDS engine is introduced to implement controlling commands. The system offers great flexibility to users by command matching and embedded compiling. In the future, a UDS over LIN may be integrated, and UDS service engine may be redesigned to support controlling multiple sunroofs simultaneously.

\section{References}

[1] Jian Wu and Surong Song: The Structure and Working Principle of Auto Electric Top Window (Auto Electric Parts, 2001).

[2] Xiaoxia Xu, Jingbin Zhao, and Yingchun Han: Automotive Sunroof Motion Mechanism Design Based on Virtual Prototype (Chinese Journal of Mechanical Engineering, 2008).

[3] ISO 14229-1:2013 - Road vehicles -- Unified diagnostic services (UDS) -- Part 1: Specification and requirements.

[4] ISO 14229-2:2013 - Road vehicles -- Unified diagnostic services (UDS) -- Part 2: Session layer services.

[5] ISO 14229-3:2012 - Road vehicles -- Unified diagnostic services (UDS) -- Part 3: Unified diagnostic services on CAN implementation (UDSonCAN).

[6] L. Zhijian and M. Tiehua: Design of CAN-Bus universal interface module based on USB (CECNet, 2011). 